\title{
Automatic data-processing equipment of moon mark of nail for verifying some experiential theory of Traditional Chinese Medicine
}

\author{
Renjie Niu*, Chenyu Fu, Zhiyong Xu and Jianyuan Huang \\ The Institute of Optics and Electronics of the Chinese Academy of Sciences, Chengdu, Sichuan, China
}

\begin{abstract}
.
BACKGROUND: Doctors who practice Traditional Chinese Medicine (TCM) diagnose using four methods - inspection, auscultation and olfaction, interrogation, and pulse feeling/palpation. The shape and shape changes of the moon marks on the nails are an important indication when judging the patient's health. There are a series of classical and experimental theories about moon marks in TCM, which does not have support from statistical data.

OBJECTIVE: To verify some experiential theories on moon mark in TCM by automatic data-processing equipment.

METHODS: This paper proposes the equipment that utilizes image processing technology to collect moon mark data of different target groups conveniently and quickly, building a database that combines this information with that gathered from the health and mental status questionnaire in each test.

RESULTS: This equipment has a simple design, a low cost, and an optimized algorithm. The practice has been proven to quickly complete automatic acquisition and preservation of key data about moon marks.

CONCLUSION: In the future, some conclusions will likely be obtained from these data; some changes of moon marks related to a special pathological change will be established with statistical methods.
\end{abstract}

Keywords: Moon mark, Traditional Chinese Medicine, real time tracking

\section{Introduction}

Medical treatment based on syndrome differentiation is the key feature of Traditional Chinese Medicine (TCM), representing the application of its theories in clinical practice. Syndrome differentiation can be considered as a process. Doctors analyze and summarize the relevant information gathered from the four diagnostic methods, inspection, auscultation and olfaction, interrogation, and pulsefeeling/palpation. Based on all symptoms and signs, doctors make a judgment of the properties of the syndrome and probe into the essence of the disease [2-5].

\footnotetext{
${ }^{*}$ Corresponding author: Renjie Niu, The Institute of Optics and Electronics of the Chinese Academy of Sciences, Lab 5, Guangdian Road, Shuang Liu, Chengdu 610209, Sichuan, China. Tel.: +86 13568907690; Fax: +86 028 85100966; E-mail: onion8203@qq.com.
} 


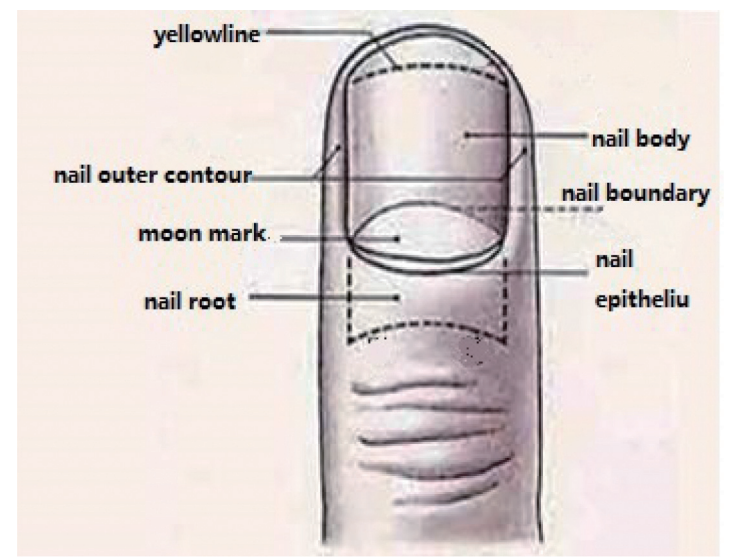

Fig. 1. Presentation of moon mark in a finger.

In TCM, a syndrome is different from a symptom. A symptom refers to the clinical manifestations of a disease, such as headache, while a syndrome is the pathological generalization of a group of closely related symptoms in the course of disease development [2-5].

There are great individual differences about different doctors' diagnostic experience from the four diagnostic methods, so various theories on TCM are not supported by statistical data. The scientific development on video provides an opportunity to solve this kind of problem.

The moon mark in the nail body is an important syndrome that can be used to judge a person's health. Figure 1 displays a moon mark.

TCM believes that the moon mark is representative of the human essence. Three characteristics of the moon mark are the number, the area, and the color. A healthy young person should have 8 to 10 moon marks with milk white color and especially clear boundaries; the area of the moon mark should account for approximately $20 \%$ of the nail. Abnormal changes in the moon mark are representative syndromes of different body parts. For example, the blackening moon mark often indicates severe heart disease, cancer, or poisoning caused by long-term drug intake or heavy metal.

A kind of special image equipment proposed in this paper tests and verifies the traditional theory of experience about moon marks through rigorous scientific statistics. An instrument is designed with automatic lighting and image acquisition. When a person puts one hand into the instrument, the month mark is automatically distinguished from the video and organized into key data. The core of the image processing algorithm is the real-time tracking of object boundaries [1], which will be elaborated on in Section 2.

\section{Design of the equipment}

The device consists of hardware subsystem and software subsystem. The hardware includes image acquisition, image analysis, and data storage, while software contains control plain interface, data recording, and image analysis.

\subsection{Hardware subsystem}

The design principles of the hardware subsystem can be summarized as a simplified structure, which consists of a mobile PC and an image acquisition box. There is a special reason for adding an image 


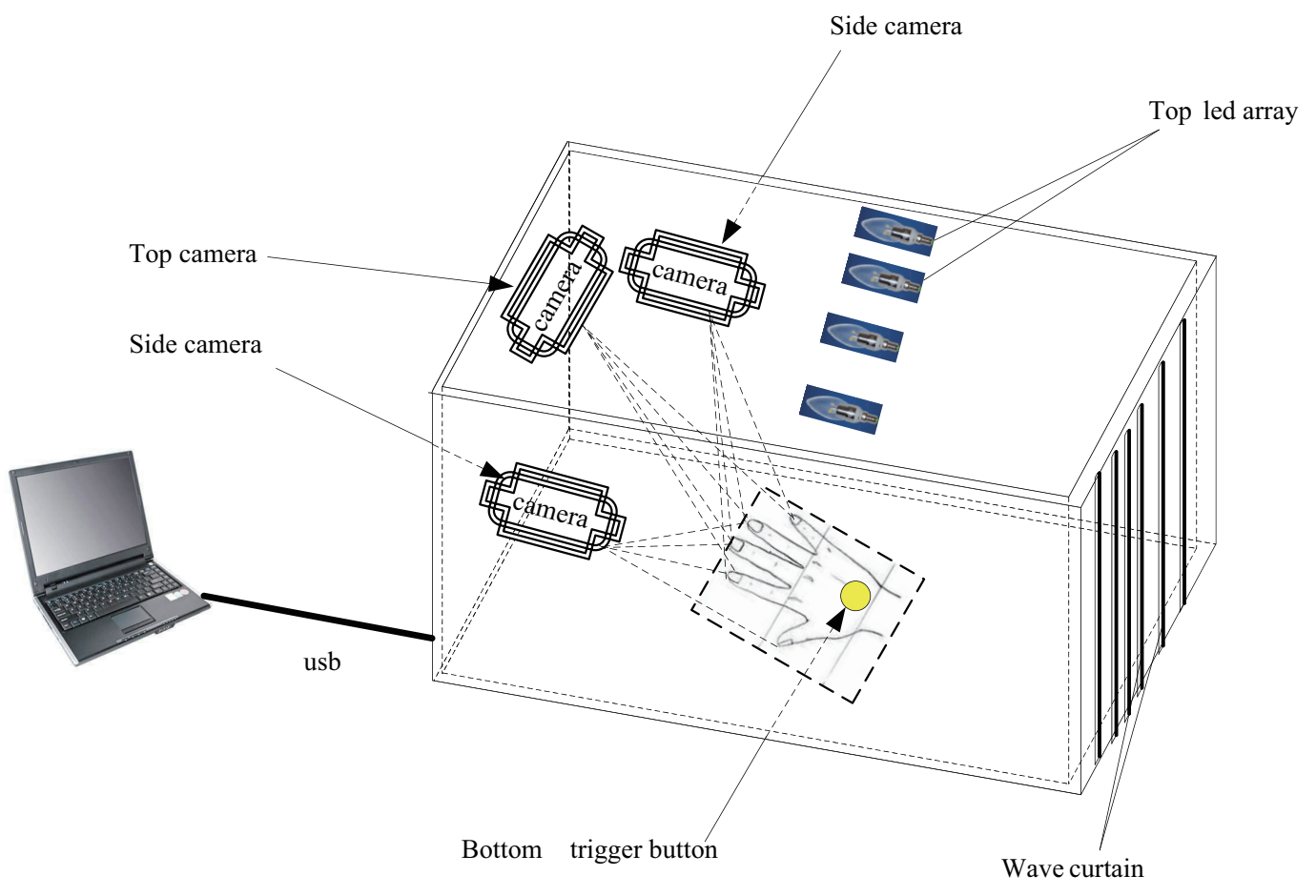

Fig. 2. The structure of image acquisition box.

acquisition box, which originates from the working principle of image processing and data mining. The soft subsystem assumes that the background is pure and invariant, the illumination condition of the box is changeless, the relative height between camera and hand remains unchanged during the testing period for ensuring effective data comparison in the coming months and years. The structure is shown in Fig. 2.

The box is composed of cameras, a light-emitting diode (LED) array, a trigger button, and a wave curtain. When a hand in the box touches the electrostatic trigger button, the led array will start to work. Meanwhile, the mobile PC gathers video from the cameras by USB. Three cameras conveniently gather positive images of each nail. As the wave curtain blocks the ambient light, if the LED array is not turned on, the average brightness of the images will be low and the software subsystem will not register a hand in the box. However, the enabled LED array substantially improves the average brightness of the images in order to analyze the details of the images.

The system requires the people's fingers apart each other slightly, so the nails will not overlap in the image. The soft can separate different finger regions for reducing the difficulty of image segmentation.

\subsection{Soft subsystem}

The software subsystem can be divided into three main steps: the initialization of the image processing program, the real-time tracking of the boundaries of the moon marks and data acquisition, the arrangement and recording of data.

The first part of the initialization image processing program is the login of the participator. The system will then acquire real-time images and wait for the activation of the LED array in the box. When the program acquires effective images, the average filter and the Susan filter immediately start. Susan filter 
is a kind of algorithms of image edge extraction for obtaining initial edges of the frame. According to the prior knowledge, the program identifies the moon mark from edge image. When a segmentation of a moon mark from a nail body does not conform to the prior knowledge, the program will tell the PC operator that there is no moon mark in the nail body and request a confirmation or segmentation again.

The PC operator can catch segmentation results of image initialization in the monitor, intervening boundary generation of moon marks simultaneously. When the correct segmentation is confirmed, the second step begins. Details of real-time tracking are presented in Section 2.2.1.

In the third step, the system has obtained an average value of the number of pixels and the RGB of the geometric center of each moon mark. Then the participator is asked to complete a simple questionnaire regarding the physical condition of each part of body and mental health, such as sleep quality, dysphoria, headache, and so on. Eventually, all data will be recorded.

\subsubsection{Real-time tracking region}

Real-time tracking of the moon mark boundaries is a key contribution for the system. It affects the average value reflected by the syndrome of moon marks. Parametric contours have been used successfully and constantly to achieve real-time performance [6,7], but there are difficulties. For this purpose, the level set methods [8] have various models and the more powerful technique $[9,10]$. But the high computational cost of the level set method has limited its application in real-time scenarios. This system, with simple background and clear object, results in the need for a simple and sufficient algorithm. In this paper, a special approach is used to create the level set method proposed in [1]. It does not solve any algorithms of partial differential equations, which reduces the computation amount compared with the optimized narrow band techniques proposed previously.

\subsubsection{Fast level set implementation}

The system uses a simple tracking strategy. In each frame, tracking results from the last frame are used as the initial curves to locate the moon mark boundaries in the current frame.

The scene from the image acquisition box is composed of the simple background $\Omega 0$ and a single moon mark $\Omega 1$. Multiple moon marks are then easily extended. Because the fingers are spread apart, the scene can be divided into several independent scenes.

To show the background $\Omega 0$ and the region of object $\Omega 1$, a level set function $\phi$ is used, $\varphi$ is negative in $\Omega 1$ and positive in $\Omega 0$. Based on our hypothesis, two lists of neighboring pixels, Outside Line and Inner Line of $\mathrm{C} 1$, as shown in Fig. 3 are defined as:

$$
\begin{aligned}
\text { Outside Line } & =\left\{x \mid \varphi(x)>0 \text { and } \exists y \in N_{4}(x) \text { such that } \mid \varphi(y)<0\right\} \\
\text { Inner Line } & =\left\{x \mid \varphi(x)<0 \text { and } \exists y \in N_{4}(x) \text { such that } \mid \varphi(y)>0\right\}
\end{aligned}
$$

In Eqs (1) and (2), N4(x) is a four connected discrete neighborhood of a pixel $\mathrm{x}$ with $\mathrm{x}$ itself being removed.

In the conventional methods of the level set, a Pareto differential evolution (PDE) should be solved either globally on the picture or locally in a narrow band to evolve the curve of the object region. The fast-level set implementations [1] are rooted on the cognitions that the curve could be evolved at pixel resolution by a simple switch of the neighboring pixels between the two lists of the Outside Line and the Inner Line.

For example, in Fig. 4, pixel A moves outward and pixel B shrinks. Compared with the curve in Fig. 3, this movement is interpreted by switching pixel A from the Outside Line to the Inner Line, and pixel B from the Inner Line to the Outside Line. By performing this action for all the pixels of the Inner Line 


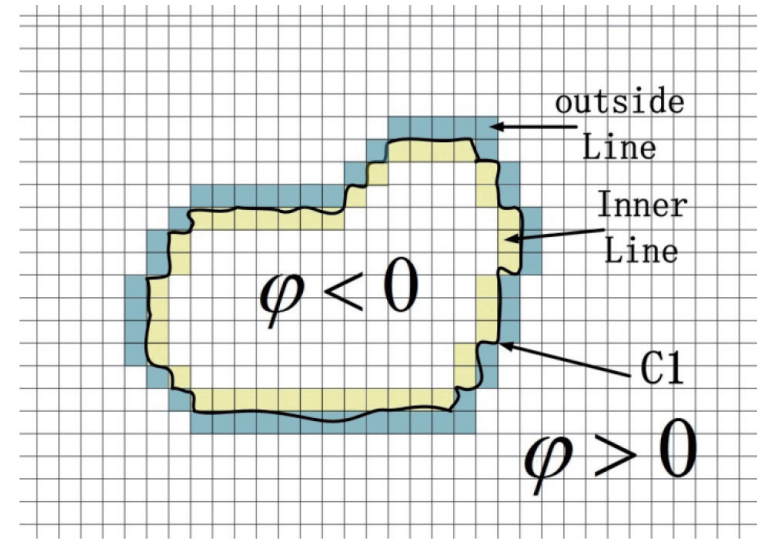

Fig. 3. Curve $\mathrm{C} 1$ and the Outside Line and the Inner Line.

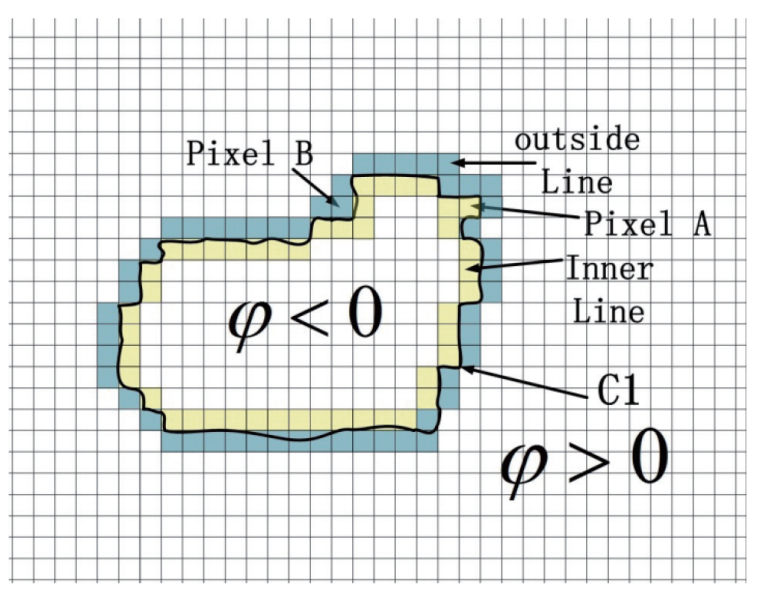

Fig. 4. Motion of the curve $\mathrm{C} 1$ by switching pixels between the two Lines.

and the Outside Line, every pixel of the curve should be transported outward or inward in the picture. The curve is represented fully, so changes in topology can be handled automatically. With this method, the soft subsystem can achieve a level-set based curve evolution at pixel resolution.

\subsubsection{Algorithm introduction}

The data structure [1] used in the algorithm is as follows:

$-\varphi$ is an array of the level set function;

- F is an array of the evolution speed;

- Two lists of neighboring pixels: Outside Line and Inner Line;

The pixels inside $\mathrm{C} 1$ but not in the Inner Line are called interior pixels, and the pixels outside $\mathrm{C} 1$ but not in the Outside Line are exterior pixels. For computer computation, $\varphi$ is defined with integers:

$$
\varphi(x)=\left\{\begin{array}{l}
3 \quad \text { if } x i \in \text { exterior pixels } \\
1 \text { if } x \in \text { Outside Line } \\
-1 \text { if } x \in \text { Inner Line } \\
-3 \text { if } x \in \text { interior pixels }
\end{array}\right.
$$

The switch of pixels between the Outside Line and the Inner Line is defined as two basic processes on the data structure.

The process of switch_in () is that a pixel ( $x \in$ Outside Line) is switched from the Outside Line to the Inner Line while moving its neighboring exterior pixels to the Outside Line. The process is formally characterized:

Switch_in ():

- Delete $x$ from the Outside Line and add it to the Inner Line. Make $\varphi(x)=-1$.

- If $\varphi(y)=3, \forall y \in N_{4}$. Add $y$ to Outside Line and make $\varphi(y)=1$.

Switch out ():

- Translate $\mathrm{x}$ from the Inner Line to the Outside Line. Make $\varphi(x)=1$.

- If $\varphi(y)=-3, \forall y \in N_{4}$. Add y to Inner Line and make $\varphi(y)=-1$. 


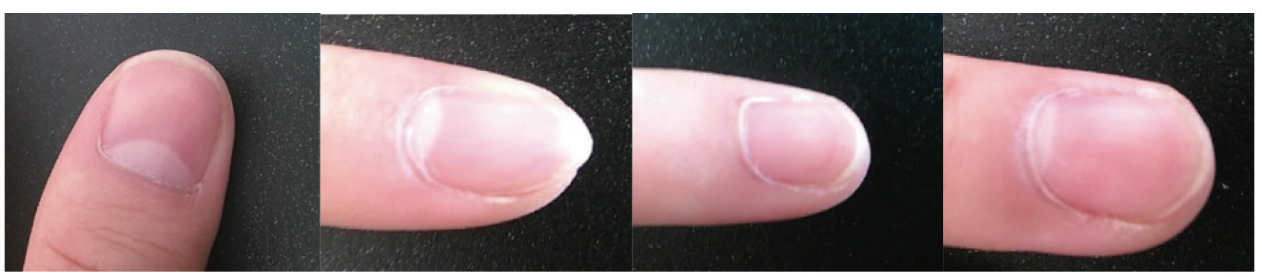

(a1) (b1) (c1) (d1)

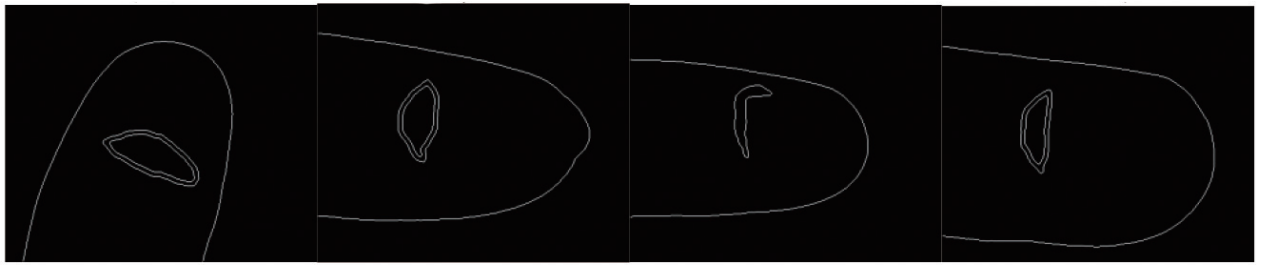

(a2) (b2) (c2) (d2)

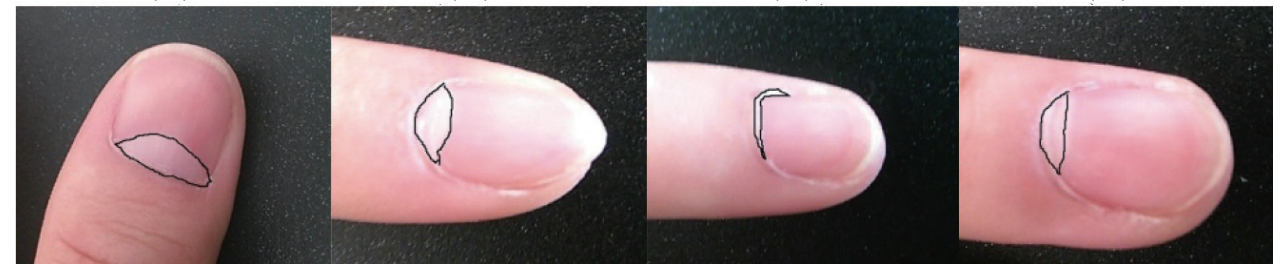

(a3)

(b3)

(c3)

(d3)

Fig. 5. Examples of real-time tracking moon mark.

To track the moon mark boundary, the soft subsystem computes the speed at all pixels in the Outside Line and the Inner Line and stores their coordinates in the array F [1].

The soft subsystem first scans the list of the Outside Line and applies the switch_in for pixels, those pixels, whose $\mathrm{F}=+1$, in the Inner Line become interior pixels. The soft subsystem then scans the list of the Inner Line and applies the switch_out for finding pixels with $F=-1$. Similarly, those pixels are translated. For the closure of iteration, a condition of stop is checked. If it works, the system will stop the evolution; otherwise, it will continue the iteration. In our device, the algorithm will stop when one of the conditions is satisfied:

- If the speed at every neighboring pixel satisfies:

$$
\begin{aligned}
& F(x) \leqslant 0 \text { if } \forall x \in \text { Outside Line } \\
& F(x) \geqslant 0 \text { if } \forall x \in \text { Inner Line }
\end{aligned}
$$

- Iterations reach the preset maximum number

If the curve is intuitively on boundary of the object, the pixels in the Outside Line will represent the background, and the pixels in the Inner Line will represent the region of object [1]. Curve convergence is completed with Condition (4) being satisfied. When the picture is noisy, curve convergence may not occur, so it is also necessary to stop the evolution.

\section{Result}

In this section, the real-time tracking results of moon marks are presented. The tests were conducted on an ordinary mobile PC with double core $2.6 \mathrm{GHz}$. The camera in the system runs at 50 frames per 
second with a frame size of $1280 \times 720$. The real-time tracking time for each frame is averaged at $0.02215 \mathrm{~s}$; under the same conditions with canny edge tracking [11], the time is averagely $0.1935 \mathrm{~s}$.

In the exam, the equipment tracks the motion of the moon marks as shown in Fig. 5. This sequence includes the marks' ordinary topological changes. This example demonstrates that the foreseeable topological changes of moon marks can be tracked with the system in real-time. The image is divided into different regions with dimensions $426 \times 350$. The original images are a1, b1, c1 and d1.The regions of the moon marks are reflected in a2, b2, c2 and d2 by the real-time tracking algorithm. A3, b3, c3 and d3 are a schematic diagram for the identification of the human eye.

It can be observed that the hand in the box is a left hand. The moon mark on the thumb, which is displayed in a1, a2 and a3, contains 5739 pixels, with an RGB of center of 226,176,188, respectively. The moon mark of the index finger, displayed in b1, b2 and b3, contains 4151 pixels with an RGB of center of 251,247,248, respectively. The moon mark of the little finger, displayed in c1, c2 and c3, contains 1566 pixels. Because this amount of pixels is extremely small, the software prompts the operator to choose whether to contain the moon mark or only the boundaries of the epitheliums. In this case, the operator chooses no moon mark. Moon mark of the middle finger, displayed in $\mathrm{d} 1, \mathrm{~d} 2$ and $\mathrm{d} 3$, contains 4151 pixels with an RGB of center of $251,247,248$, respectively.

\section{Conclusion}

This equipment has the advantages of a simple design, a low cost and an optimized algorithm; the exclusive image acquisition box greatly eliminates the random error from manual operation and the environment in order to enhance image contrast. It can utilize image processing technology to collect moon mark data of different target groups conveniently and quickly, then building a database. This database is combined with the health and mental status questionnaire of each participant in the experiment. With possibility of expanding the database in the future, some conclusions will hopefully be obtained from these data; some of the changes in moon marks and their relations to pathology can be established in statistics.

\section{References}

[1] Yonggang Shi and Karl W.C., Real-time tracking using level sets, Computer Vision and Pattern Recognition, 2005. CVPR 2005. IEEE Computer Society Conference, vol. 2. p. 34-41.

[2] Wang Yongyan, Liu Baoyan and Xie Yanming, Establishing appraisal mechanism of clinic studies in Traditional Chinese Medicine with proofing Medicine Methodology, Chinese Journal of basic Traditional Chinese Medicine, 2003. vol. 9. NO. 3. p. 17-24.

[3] Luo man, The spread of western medicine in china and collision between western medicine and Traditional Chinese Medicine, Medicine and Philosophy, 2009. vol. 30. NO. 7. p. 60-63.

[4] Ding jianguo, The potential logical conflicts between the Traditional Chinese Medicine, Medicine and Philosophy, 2009. vol. 30. NO. 4. p. 66-67.

[5] Zhong chaozhong, View Western Medicine through Chinese Medicine, Medicine and Philosophy, 2010. vol. 31. NO. 5. p. 53-55.

[6] M. Isard and A. Blake, ICONDENSATION: Unifying low level and high-level tracking in a stochastic framework, Proc. ECCV. 1998. P. 767-781.

[7] D. Comaniciu, V. Ramesh, and P. Meer, Kernel-based object tracking, IEEE Trans. Pattern Anal. Machine Intell. May 2003, vol. 25 , no. 5, p. 564-577.

[8] S. Osher and J. Sethian, Fronts propagation with curvature dependent speed: algorithms based on Hamilton-Jacobi formulations, Journal of computational physics, 1988, vol. 79, p. 12-49. 
[9] M. Bertalmio, G. Sapiro, and G. Randall, Morphing activecontours: A geometric approach to topology-independent image segmentation and tracking, 1998, p. 318-322.

[10] S. Besson, M. Barlaud, and G. Aubert, Detection and tracking of moving objects using a new level set based method, Proc. ICPR, Sept 2000, vol. 3, p. 1100-1105.

[11] John Canny. A Computational Approach to Edge Detection, IEEE Transactions on pattern analysis and machine intelligence, November 1986, vol. pami-8, NO. 6. 